\title{
Transmural Versus Nontransmural In Situ Electrical Impedance Spectrum for Healthy, Ischemic, and Healed Myocardium
}

\author{
Yolocuauhtli Salazar*, Ramon Bragos, Member, IEEE, Oscar Casas, Associate Member, IEEE, Juan Cinca, and
} Javier Rosell, Member, IEEE

\begin{abstract}
Electrical properties of myocardial tissue are anisotropic due to the complex structure of the myocardial fiber orientation and the distribution of gap junctions. For this reason, measured myocardial impedance may differ depending on the current distribution and direction with respect to myocardial fiber orientation and, consequently, according to the measurement method. The objective of this study is to compare the specific impedance spectra of the myocardium measured using two different methods. One method consisted of transmural measurements using an intracavitary catheter and the other method consisted of nontransmural measurements using a four-needle probe inserted into the epicardium. Using both methods, we provide the in situ specific impedance spectrum (magnitude and phase angle) of normal, ischemic, and infarcted pig myocardium tissue from $1 \mathrm{kHz}$ to $1 \mathrm{MHz}$. Magnitude spectra showed no significant differences between the measurement techniques. However, the phase angle spectra showed significant differences for normal and ischemic tissues according to the measurement technique. The main difference is encountered after $60 \mathrm{~min}$ of acute ischemia in the phase angle spectrum. Healed myocardial tissue showed a small and flat phase angle spectrum in both methods due to the low content of cells in the transmural infarct scar. In conclusion, both transmural and nontransmural measurements of phase angle spectrum allow the differentiation among normal, ischemic, and infarcted tissue.
\end{abstract}

Index Terms-Anisotropy, electrical impedance, intracavitary catheter, myocardial impedivity, resistivity.

\section{INTRODUCTION}

$\mathbf{T}$ HE measured impedance $(Z)$ is a complex number defined as the ratio between measured voltage $(V)$ and total current flow $(I)$. For a homogeneous and isotropic object, it is a function of its electrical properties (conductivity and permittivity), but it also depends on geometric factors. The relation between $Z$ and these parameters is

$$
\begin{aligned}
Z & =k \frac{1}{\sigma+j \omega \varepsilon_{0} \varepsilon_{r}}=k \frac{1}{\sigma^{*}}=k \rho^{*} \\
& =k(r+j x)=k\left|\rho^{*}\right|_{\text {Phase }\left(\rho^{*}\right)}
\end{aligned}
$$

Manuscript received July 18, 2003; revised February 1, 2004. This work was supported by MCYT and FEDER, Project SAF-2001-1660. The work of Y Salazar was supported by a scholarship through CONACYT (Mexico). Asterisk indicates corresponding author.

*Y. Salazar is with the Department of Electronic Engineering, Universidad Politécnica de Cataluña, Barcelona, Spain (e-mail: ysalazar@eel.upc.es).

R. Bragos, O. Casas, and J. Rosell are with the Department of Electronic Engineering, Universidad Politécnica de Cataluña, Barcelona 08034, Spain.

J. Cinca is with the Department of Cardiology, Hospital de la Santa Creu y

Sant Pau, 08025 Barcelona, Spain.

Digital Object Identifier 10.1109/TBME.2004.828030 where $k$ is the "cell factor" $\left(\mathrm{m}^{-1}\right)$ and depends on the geometry of the electrodes and the object, $\sigma$ is the electrical conductivity $(\mathrm{S} / \mathrm{m}), j$ is the imaginary symbol, $\omega$ is the frequency, $\varepsilon_{0}$ is the vacuum permittivity, and $\varepsilon_{\mathrm{r}}$ is the relative permittivity. Instead of using the complex conductivity $\left(\sigma^{*}=\sigma+j w \varepsilon_{0} \varepsilon_{\mathrm{r}}\right)$, we could define the complex impedivity $\left(\rho^{*}=r+j x\right)$. The real part of the impedivity is the resistivity and the imaginary part the reactivity. Impedance measurements were historically given as resistivity because the imaginary part was small and was neglected. This is a correct approximation, especially at low frequencies (1 kHz and below). However, at higher frequencies, the imaginary part increases and the impedivity must be considered as a complex number. As the term impedivity is not commonly used, we used the term specific impedance and the polar representation (magnitude and phase angle).

Previous equations are valid only for a homogenous and isotropic object. Myocardial tissue could be considered homogeneous but it is highly anisotropic due to the complex structure of the fiber orientation. At an intermediate scale $(100-1000 \mu \mathrm{m})$, the elongated structure and the parallel alignment of the muscle cells (with a typical length of $100 \mu \mathrm{m}$ ) result in a bidirectional electrical anisotropy: longitudinal and transversal to the direction of alignment (fiber direction). At the macroscopic level $(1-10 \mathrm{~mm})$, a gradual change in fiber direction over more than $120^{\circ}$ is found, going from epicardium to endocardium (about $20^{\circ}$ over the first tenth of wall thickness) [1]. This structure makes it difficult to obtain the longitudinal and transversal specific impedance from the measurements made with four intramural needle electrodes [2]. Some authors [3], [4] have reported longitudinal and transversal resistivities, and also in [5] a special probe and an analytical method for separating transversal and longitudinal resistivities were introduced, but other authors have been unable to differentiate the anisotropy [6], [2]. Given the difficulty of separating the two components, especially for in situ measurements, we did not use this distinction a priori.

Knowledge of the specific electrical impedance spectrum of myocardial tissues is important in simulations involving propagation of electrical signals [7]-[9] and especially in the development of new techniques using impedance for diagnosis and monitoring. Recently, new applications based on the use of a catheter to measure impedance have been proposed: for example, the location of the infarction border zone [10] and the online monitoring of myocardium tissue state [11] and lesion dimensions [12], [13] during ablation procedures. 


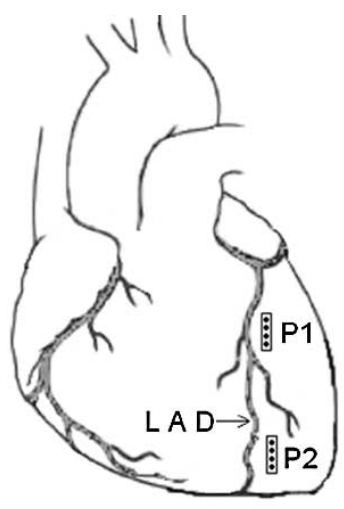

(a)

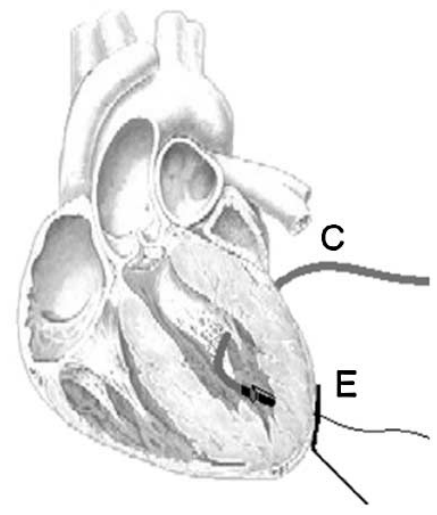

(b)
Fig. 1. Schematic representation of the two experimental procedures. (a) Nontransmural method showing the approximate locations for the four-needle probes, for healthy (P1) and ischemic (P2) areas. (b) Transmural method showing the catheter (C) inserted through the posterior left ventricle with the tip at the measurement location in the endocardial surface in front of the reference electrode $(\mathrm{E})$ located on the epicardium.

In commercial radio frequency (RF) ablation equipment, electrical impedance is already used to determine the electrode-tissue contact [8]. During RF ablation procedures, the position of the catheter is determined by fluoroscopy and electrocardiographic recordings. The study of the impedance technique is of interest as an additional method for ensuring the correct position of the electrode tip, because the efficacy of catheter ablation depends on the accurate identification of the site of origin of the arrhythmia and the correct positioning of the catheter for ablation. The use of impedance measurements could reduce the length of the procedure and the exposure to radiation.

There is much published myocardial impedance data measured with four intramural needles but very little [10], [11] using a transcatheter technique. Due to myocardial anisotropy, the use of data obtained with one technique may be not valid for the other. In this study, we only considered in situ measurements made using these two methods: the widely accepted four equally spaced needle electrodes inserted on the epicardium (nontransmural measurement) and the use of an intracavitary catheter (transmural measurement). See Fig. 1 for a schematic representation of both methods.

We have shown in previous studies that there are significant differences in the electrical impedance of the myocardium tissue depending on its pathophysiological state. For example, by measuring the impedance spectrum with four-needle electrodes on the epicardium, it is possible to monitor the evolving change of the tissue during ischemia [14]. Also, using a transmural method, healthy and healed infarcted tissue can be differentiated [11].

This paper aims to provide a complete comparison of in situ specific impedance spectra for normal, ischemic, and healed myocardium using transmural and nontransmural measurements. Nontransmural results for healthy and ischemic myocardium came from a new group of animals and healed results were obtained from a previous experiment [15]. For transmural impedances, we used the same group of animals as in [11], incorporating the ischemic results not published in that paper. We also evaluate the cell factor for the transmural method in order to obtain the specific impedance $(\Omega \cdot \mathrm{cm})$, thus

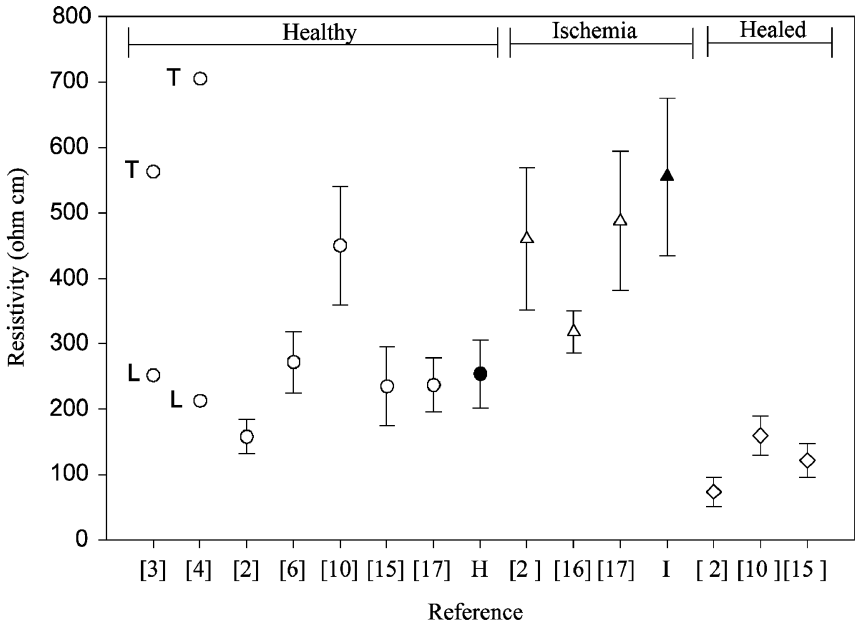

Fig. 2. In vivo nontransmural electrical resistivities at $1 \mathrm{kHz}$ for healthy (circle), ischemic (triangle), and healed (diamond) myocardium. Results from Group I (solid) compared with previous studies (open). All data are represented as mean \pm STD except for [3] and [4] that shows mean values for longitudinal (L) and transversal (T) resistivities.

allowing a comparison between the spectra obtained with both techniques. The results will contribute to better modeling and the optimization of new in vivo measurement techniques to characterize myocardial tissue state.

\section{Previous Studies}

This section reviews previously published myocardial impedance data. We selected only those studies that use in vivo and in situ measurements. Data are classified depending on the measurement method: nontransmural using four electrodes (at $1 \mathrm{kHz}$ and multifrequency) and transmural using a catheter. Data are expressed as mean \pm standard deviation.

\section{A. Nontransmural at $1 \mathrm{kHz}$}

Measurement of resistivity at $1 \mathrm{kHz}$ with four-needle electrodes inserted into the epicardium is the method used in most of the previous studies. Fig. 2 shows the published resistivities obtained at $1 \mathrm{kHz}$ for different tissue states: healthy, healed, and ischemic myocardium.

Rush et al. [3] reported myocardial resistivities in dogs (the real part of the specific impedance) of $563 \Omega \cdot \mathrm{cm}$ perpendicular to myocardial muscle fiber orientation and $252 \Omega \cdot \mathrm{cm}$ parallel to the fibers. Roberts and Scher [4] reported (also in dogs) gross myocardial resistivities of $213 \Omega \cdot \mathrm{cm}$ parallel to fibers and $705 \Omega \cdot \mathrm{cm}$ perpendicular to fiber orientation. Ellenby et al. [16] report a mean baseline resistivity measurement made in vivo of $318.5 \pm 32.5 \Omega \cdot \mathrm{cm}$ (six dogs).

More recently, Fallert et al. [2] studied the evolution of infarction after coronary occlusion in sheep. They found that the average baseline specific impedance at $1 \mathrm{kHz}$ measured before infarction in nine animals was $158 \pm 26 \Omega \cdot \mathrm{cm}, 60 \mathrm{~min}$ after coronary occlusion it was $460 \pm 109 \Omega \cdot \mathrm{cm}$, and six-week-old infarctions revealed average values of $74 \pm 22 \Omega \cdot \mathrm{cm}$. The differences between transverse and longitudinal specific impedance in normal hearts showed a small but statistically significant difference $(170 \pm 33$ versus $151 \pm 25 \Omega \cdot \mathrm{cm})$.

Schwartzman et al. [10] used four electrodes on ovine models to obtain the electrical impedance properties of healthy and 
chronically infarcted ventricular myocardium. With epicardial measurements performed in vivo, they confirmed that marked differences in impedance exist between healthy $(450 \pm 90 \Omega \cdot \mathrm{cm})$ and chronically infarcted myocardium $(160 \pm 30 \Omega \cdot \mathrm{cm})$.

Cinca et al. [17] measured the evolution during ischemia of tissue resistivity in 26 pigs at $1 \mathrm{kHz}$ using a four-electrode probe (length $=5 \mathrm{~mm}$, diameter $=0.4 \mathrm{~mm}$ interelectrode distance $=2.5 \mathrm{~mm}$ ). They reported a baseline myocardial resistivity of $237 \pm 41 \Omega \cdot \mathrm{cm}$, while after $60 \mathrm{~min}$ of acute ischemia the resistivity was $488 \pm 100 \Omega \cdot \mathrm{cm}$.

\section{B. Nontransmural Multifrequency}

Steendijk et al. [5] used a four-electrode method to measure local myocardial resistivity in two orthogonal directions in anesthetized open chest dogs. Transversal and longitudinal resistivities were estimated applying an analytical correction to orthogonal measurements. The results are given in the frequency range 5-60 kHz. Longitudinal resistivity decreased from $313 \pm 49$ at $5 \mathrm{kHz}$ to $212 \pm 32 \Omega \cdot \mathrm{cm}$ at $60 \mathrm{kHz}$ and transversal resistivity decreased from $487 \pm 49$ at $5 \mathrm{kHz}$ to $378 \pm 53 \Omega \cdot \mathrm{cm}$ at $60 \mathrm{kHz}$.

Tsai et al. [6] used a four-electrode probe (length $=4.5 \mathrm{~mm}$, diameter $=0.41 \mathrm{~mm}$. interelectrode distance $=1.5 \mathrm{~mm}$ ) to measure myocardial swine resistivity in vivo in two directions at eight frequencies from $1 \mathrm{~Hz}$ to $1 \mathrm{MHz}$ (eight pigs). They showed that the mean measured myocardial resistivity was $272 \pm 47 \Omega \cdot \mathrm{cm}$ at $1 \mathrm{~Hz}$ which came down to $166 \pm 15 \Omega \cdot \mathrm{cm}$ at $1 \mathrm{MHz}$. Their results showed no significant difference in orthogonal directions.

\section{Transmural}

Schwartzman et al. [10] measured the ovine myocardial impedance at $550 \mathrm{kHz}$ in four animals using a catheter inserted percutaneously. The measurement was between the catheter tip and an external cutaneous electrode (unipolar method). Results show that it is possible to distinguish between healthy $(345 \pm 15 \Omega)$ and chronically infarcted (healed) myocardium $(240 \pm 10 \Omega)$.

In conclusion, with the four-needle electrode method, the differentiation between tissues has been done mainly using the impedance magnitude at $1 \mathrm{kHz}$. Multifrequency measurements were oriented to obtain impedance spectra for normal tissue in longitudinal and transversal directions.

The transmural measurements from Schwartzman et al. [10] showed that it is possible to distinguish between healthy and healed myocardium but only using the impedance magnitude at one frequency $(550 \mathrm{kHz})$.

It is necessary to know if the impedance magnitude at $1 \mathrm{kHz}$ is the best selection to distinguish between myocardium tissue states and, most important, to know if the nontransmural myocardial spectra obtained with the four-needle electrode method are the same as the ones obtained using a transmural method based on a catheter.

\section{METHODS}

\section{A. Experimental Methods}

For all of the experiments, data were obtained from open chest pigs anesthetized with $\alpha$-chloralose under mechanical ventilation as in [15]. The thorax was opened through a midsternotomy and the pericardium was gently detached. The pigs were handled in accordance with the European Community rules on animal research. The studies were approved by the ethics committee of the hospital.

A Hewlett-Packard 4192A impedance analyzer, with a custom front-end amplifier, was used to measure the impedance spectrum from $1 \mathrm{kHz}$ to $1 \mathrm{MHz}$ at eight logarithmically equally spaced frequencies per decade. The front-end was a differential amplifier designed to obtain a high common-mode rejection ratio (better than $72 \mathrm{~dB}$ up to $1 \mathrm{MHz}$ ). We used 15-cm coaxial cables between the front-end and the electrodes to reduce crosstalk. Voltage detection cables used driven shields to minimize input capacitance.

1) Four-Electrode Nontransmural Measurements: The normal and ischemic nontransmural impedance data were obtained from seven pigs (Group I: $25-30 \mathrm{~kg}$ ). To produce an area of myocardial ischemia, the left anterior descending (LAD) coronary artery was occluded below the first diagonal branch by means of a ligature. The evolving changes in myocardial impedance were measured during four hours at the center of the ischemic area and at a remote normal myocardial site with two probes that were sutured to the epicardium. The probes were sutured trying to maintain the same position and orientation (parallel to the LAD artery) in all of the animals. The appropriate position of each probe was verified at the end of the study using fluoresceine.

Each impedance probe consisted of a linear array of four platinum needles ( $0.4 \mathrm{~mm}$ in diameter) separated by an interelectrode distance of $2.5 \mathrm{~mm}$. The needles were inserted into a rigid flat plastic fixture that assured the perpendicularity with respect to the myocardium surface. The length of the platinum needles to be inserted on the myocardium was $5 \mathrm{~mm}$.

Impedance of healed infarction was measured on a different group of seven pigs (Group II: $25-30 \mathrm{~kg}$ ). This group is the same as group 2 in [15]. The healed infarction area was produced by a permanent ligature of the LAD through a left lateral thoracotomy one month before the open chest measurements. We used a shorter Platinum-Iridium probe $(3.5 \mathrm{~mm}$ long, $0.2 \mathrm{~mm}$ diameter, $2.5 \mathrm{~mm}$ interelectrode distance) to facilitate insertion into the healed myocardium. After completion of the in vivo study, the heart was removed and a histological examination was performed on infarcted and normal measurement sites.

2) Transmural Measurements: In situ transmural impedance data were obtained from another group of four pigs (Group III: 15-20 kg). This group is the same used in [11] to obtain the normal and healed myocardium impedances. As in the previous experiment, an infarct was previously produced (two months before) by a permanent occlusion of the LAD artery below the first diagonal branch. During the open chest experiment, a second ligature of the LAD coronary artery was produced over the first diagonal branch to produce a new area of acute ischemia.

Transmural impedance spectra were measured employing a custom intracavitary catheter (constructed by C. R. BARD). The catheter was bipolar, with a standard platinum tip and a proximal electrode separated by $2 \mathrm{~mm}$ [18]. The reference electrode $(20 \times 20 \times 0.2 \mathrm{~mm}$ platinum foil $)$ was placed on the surface of the left anterior ventricular region opposite to the catheter tip. A three-electrode measurement method was used; a subthreshold 
electrical current was applied between the tip and the reference electrode and the voltage was measured between proximal and reference electrodes.

The catheter was introduced into the left ventricle through the posterior ventricular wall with the help of a 10-cm-long needle with a thread secured to the catheter tip. The catheter was pulled from the posterior wall to the selected location of the anterior ventricular wall. The thread was passed through a pore in the center of the epicardial platinum electrode to ensure alignment between the catheter and the reference electrode. After measuring the impedance, the thread was cut and left in place to mark the measurement site. The catheter tip was positioned in both healthy and healed infarcted sites. In each pig, we explored at least seven sites extending from the scar to the surrounding healthy myocardium.

The ischemic impedance spectrum was measured at two sites for each animal. At the first site, we measured one spectrum every 2 min for 60 min after the occlusion of the LAD coronary artery. The second site was selected in a normal area and measurements were performed postmortem for $60 \mathrm{~min}$. Animal temperature was maintained with a thermal pad.

The location of measurement sites was verified using fluoresceine and histological examination. We classified the sites into four categories: normal, ischemic, healed, and infarct border.

To allow for a comparison between transmural and nontransmural measurements, we converted the transmural impedance results into specific impedance $(\Omega \cdot \mathrm{cm}$ and phase angle) using the method described in the next section.

\section{B. Evaluation of Cell Factors}

The $k$ value for nontransmural measurements was determined by measuring the impedance of a $0.9 \% \mathrm{NaCl}$ solution at $25^{\circ} \mathrm{C}$ using the same four-electrode needles and measurement equipment that was used for the in situ studies.

The geometric factor $(k)$ for transmural measurements was calculated from simulations. We constructed two finite element heart models (one for a normal heart and the other for a heart with a scar) including the catheter and the reference electrode in the same positions as in the experiment. The myocardium thickness used for the model was the mean value obtained from the histological analysis of each animal $(4 \mathrm{~mm}$ for the scar and $12 \mathrm{~mm}$ for healthy and ischemic tissue). An isotropic and uniform resistivity was assigned to all of the tissue and we calculated the measured impedance. $k$ was calculated as the ratio between the measured impedance and the resistivity.

The limitation of this method is that the cell factor is only constant for an object with homogeneous and isotropic specific impedance. However, for a nonisotropic object, the cell factor also depends on the distribution and direction of current lines inside the object. As the myocardium impedance has a strong anisotropy, the estimated specific impedance magnitude has limited accuracy. It must be emphasized that the phase angle of the specific impedance does not depend on the cell factor $(k)$.

\section{Data Analysis}

Cardiac related variations in impedance were reduced by averaging five consecutive impedance spectra. To reduce systematic gain and phase errors, each impedance spectrum was
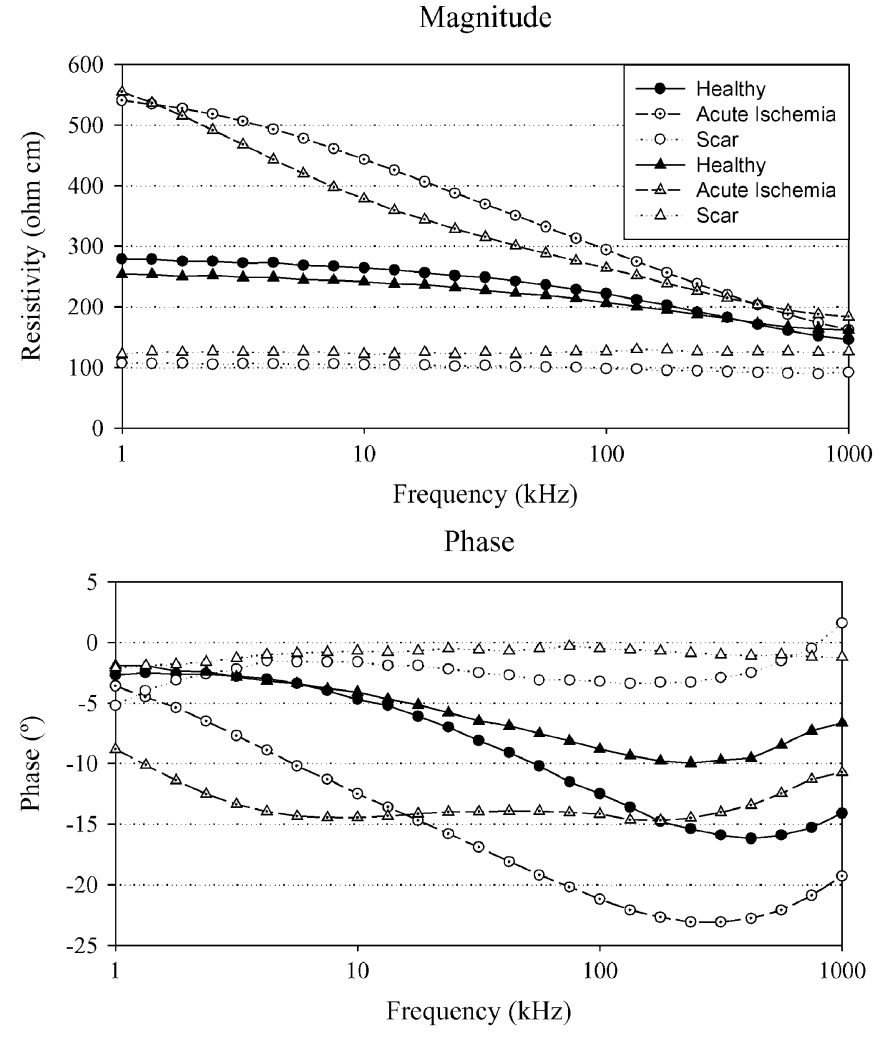

Fig. 3. Mean specific impedance (magnitude and phase angle) for healthy, ischemic, and healed myocardium measured using two methods: nontransmural using a four-needle probe inserted into the epicardium (triangles) and transmural using a three-electrode transcatheter method (circles). Numerical values for the mean and the STD for a subset of frequencies are given in Tables I and II.

calibrated using a three-reference method [19]. The three reference spectra were acquired by measuring saline solutions of known conductivity. Differences in the impedance values were assessed by Student's t-test. For each statistical test, a $p$ value $<0.05$ was considered significant.

\section{RESULTS}

Nontransmural impedance was measured at seven healthy sites (group I), seven ischemic sites (group I), and seven healed infarcted sites (group II). In group II, all seven animals developed a transmural healed myocardial infarction with a thickness of 3-4 $\mathrm{mm}$ and a subendocardial surviving cell layer of $0.2-0.3 \mathrm{~mm}$. The infarcted tissue was composed by fibroblast, collagen fibers, and a few capillary vessels. The seven "healthy" sites measured in group II were not considered because the heart could not be considered normal having a one-month-old infarction.

Transmural impedance was measured at 37 sites: 17 in the infarcted area, 9 in the normal myocardium, 5 in the infarct border, and 6 in the normal myocardium during acute ischemia (one of the animals died prematurely). All four pigs (group III) developed a healed anterior transmural myocardial infarction with a thickness of about $4 \mathrm{~mm}$ and a subendocardial surviving cell layer of $0.1-0.3 \mathrm{~mm}$.

Fig. 3 compares the mean value of specific impedance spectra for transmural and nontransmural myocardial measurements for healthy myocardium, acute ischemia, and healed myocardium. 
TABLE I

SPECIFIC IMPEDANCE MAGNITUdE $(\Omega \cdot \mathrm{CM})$ FOR MYOCARDIAL TISSUE IN Function of the Measurement Method and the Tissue State. VALUES ARE MEAN \pm STANDARD DEVIATION

\begin{tabular}{lrcc}
\hline & Freq $(\mathrm{kHz})$ & $\begin{array}{c}\text { Non- } \\
\text { transmural }\end{array}$ & Transmural \\
\hline Healthy & 1.00 & $254 \pm 52$ & $279 \pm 81$ \\
Myocardium & 3.16 & $249 \pm 51$ & $273 \pm 77$ \\
& 10.00 & $242 \pm 48$ & $264 \pm 75$ \\
& 31.60 & $227 \pm 44$ & $248 \pm 68$ \\
& 100.00 & $206 \pm 40$ & $222 \pm 59$ \\
& 316.00 & $181 \pm 37$ & $182 \pm 48$ \\
& 1000.00 & $161 \pm 32$ & $146 \pm 41$ \\
\hline Acute Ischemia & 1.00 & $555 \pm 120$ & $540 \pm 260$ \\
(1 hour) & 3.16 & $468 \pm 68$ & $506 \pm 229$ \\
& 10.00 & $378 \pm 48$ & $443 \pm 184$ \\
& 31.60 & $315 \pm 43$ & $369 \pm 142$ \\
& 100.00 & $264 \pm 38$ & $294 \pm 112$ \\
& 316.00 & $214 \pm 34$ & $220 \pm 90$ \\
& 1000.00 & $184 \pm 30$ & $162 \pm 75$ \\
\hline Healed & 1.00 & $122 \pm 26$ & $107 \pm 34$ \\
Myocardium & 3.16 & $125 \pm 27$ & $107 \pm 33$ \\
(scar) & 10.00 & $122 \pm 27$ & $104 \pm 31$ \\
& 31.60 & $125 \pm 24$ & $103 \pm 30$ \\
& 100.00 & $126 \pm 25$ & $98 \pm 27$ \\
& 316.00 & $125 \pm 24$ & $93 \pm 26$ \\
& 1000.00 & $126 \pm 24$ & $92 \pm 27$ \\
\hline & & &
\end{tabular}

TABLE II

Phase Angle $\left({ }^{\circ}\right)$ For Myocardial Tissue as a Function OF THE Measurement Method and the Tissue State. Values ARE Given as MEAN \pm Standard DEVIaTION

\begin{tabular}{crrr}
\hline & & \multicolumn{1}{l}{ Non- } & \\
& Freq $(\mathrm{kHz})$ & \multicolumn{1}{c}{ transmural } & \multicolumn{1}{c}{ Transmural } \\
\hline Healthy & 1.00 & $-1.9 \pm 1.7$ & $-2.7 \pm 0.8$ \\
Myocardium & 3.16 & $-2.9 \pm 1.4$ & $-2.8 \pm 0.7$ \\
& 10.00 & $-4.1 \pm 1.3$ & $-4.7 \pm 0.8$ \\
& 31.60 & $-6.5 \pm 1.0$ & $-8.1 \pm 1.0$ \\
& 100.00 & $-8.8 \pm 0.7$ & $-12.5 \pm 1.5$ \\
& 316.00 & $-9.7 \pm 1.7$ & $-15.9 \pm 2.4$ \\
& 1000.00 & $-6.6 \pm 4.2$ & $-14.1 \pm 8.1$ \\
\hline Acute Ischemia & 1.00 & $-8.8 \pm 4.4$ & $-3.6 \pm 3.3$ \\
(1 hour) & 3.16 & $-13.4 \pm 6.2$ & $-7.7 \pm 3.8$ \\
& 10.00 & $-14.5 \pm 4.9$ & $-12.5 \pm 3.4$ \\
& 31.60 & $-14.0 \pm 3.1$ & $-16.9 \pm 2.7$ \\
Healed & 100.00 & $-14.2 \pm 2.3$ & $-21.2 \pm 3.5$ \\
Myocardium & 316.00 & $-14.0 \pm 1.9$ & $-23.1 \pm 4.3$ \\
(Scar) & 1000.00 & $-10.7 \pm 2.6$ & $-19.3 \pm 5.1$ \\
\hline & 1.00 & $-2.1 \pm 1.7$ & $-5.2 \pm 1.0$ \\
& 3.16 & $-1.3 \pm 1.3$ & $-2.2 \pm 0.8$ \\
& 10.00 & $-0.7 \pm 1.3$ & $-1.6 \pm 1.1$ \\
& 31.60 & $-0.6 \pm 1.2$ & $-2.5 \pm 1.1$ \\
& 100.00 & $-0.5 \pm 1.5$ & $-3.2 \pm 1.3$ \\
& 316.00 & $-1.0 \pm 1.3$ & $-2.9 \pm 3.2$ \\
& 1000.00 & $-1.2 \pm 1.2$ & $-1.6 \pm 12.7$ \\
\hline
\end{tabular}

For clarity, the means and standard deviations are given in Tables I and II at representative frequencies.

\section{A. Comparison Between Techniques}

For healthy myocardium, there are no significant $(p>0.4)$ differences between transmural and nontransmural impedance magnitudes over the whole spectrum. In the phase angle spectrum, over $10 \mathrm{kHz}$, the difference between mean values increases, reaching a maximum at $750 \mathrm{kHz}$ of $8^{\circ}$ (nontransmural: $-7.3 \pm 3.8^{\circ}$ versus transmural: $-15.3 \pm 5.9^{\circ}, \mathrm{p}=0.15$ ).

The phase spectrum for myocardial tissue after $60 \mathrm{~min}$ of acute ischemia shows a significant difference between the measurement methods. Nontransmural specific impedance shows a low-frequency relaxation $\left(-14.5 \pm 4.9^{\circ}\right.$ at $\left.10 \mathrm{kHz}\right)$ and this low phase is maintained up to $300 \mathrm{kHz}$, while transmural specific impedance only shows a dominant high-frequency relaxation. The biggest difference (Table II) is at $316 \mathrm{kHz}$ (nontransmural: $-14 \pm 1.9^{\circ}$ versus transmural: $\left.-23.1 \pm 4.3^{\circ}, \mathrm{p}<0.001\right)$.

The specific impedance of healed myocardium shows no significant differences in magnitude and phase angle between the two measurement techniques (Tables I and II).

\section{B. Differentiation Between Tissue States}

Using nontransmural measurements, acute ischemic myocardium shows a mean increase of $118 \%$ in the impedance magnitude at $1 \mathrm{kHz}$ with respect to healthy myocardium $(p<0.0001)$, while the healed myocardium shows a $52 \%$ decrease $(p<0.0001)$.

For transmural measurements, we obtain changes of the same magnitude (94\%) between acute ischemia and healthy tissue, but with a lower significance $(p=0.056)$. The scar impedance shows a decrease of $62 \%$ with respect to healthy tissue $(p<$ 0.0001).

The phase angle spectrum provides better tissue differentiation than the impedance magnitude spectrum using transmural as well as nontransmural measurements. The frequencies that give the most significant differences between healthy and healed tissue are $237 \mathrm{kHz}$ for nontransmural measurements $(\mathrm{p}<1.3 \exp -8)$ and $178 \mathrm{kHz}$ for transmural measurements $(\mathrm{p}<4.1 \exp -15)$. To differentiate between healthy and ischemic tissue, the optimal frequencies are $56 \mathrm{kHz}$ for nontransmural measurements $(\mathrm{p}<4.6 \exp -5)$ and $42 \mathrm{kHz}$ for transmural measurements $(\mathrm{p}<2.5 \exp -4)$.

\section{DISCUSSION}

Fig. 2 compares previous studies with our results for nontransmural resistivity at $1 \mathrm{kHz}$ for the three tissue states. For healthy tissue, our results are in agreement with previously published in vivo data measured following the longitudinal fiber direction [3], [4] or in arbitrary directions [2], [6], [17]. The biggest difference, in longitudinal or arbitrary directions, is with the value reported for healthy tissue by Schwartzman et al. [10] but, as stated in the paper, this value could be attributed to ischemic tissue. Ischemic tissue resistivity is also in good agreement with that reported in [2] and [17] but is higher than that given in [16]. The differences in the mean resistivity values at 1 $\mathrm{kHz}$ reported by different authors and the high standard deviations, especially for ischemic tissue, must be emphasized. This dispersion implies a strong limitation in the use of the resistivity as an estimator for tissue characterization. 
Histological analysis of healed infarcted myocardium revealed that surviving cells form a thin layer $(0.1-0.3 \mathrm{~mm})$ on the endocardium and the rest of the wall is composed of fibroblasts and collagen. Theoretically, the absence of cells in the major part of the wall will produce no relaxation phenomena (phase angle equal to zero and constant magnitude) at the frequency range analyzed. This is confirmed by our results and previously published work by Schwartzman et al. [10], using in vitro measurements between $1 \mathrm{kHz}$ and $10 \mathrm{MHz}$, and by Fallert et al. [2], using in vivo data at 1,5 , and $15 \mathrm{kHz}$. For this reason, the measured magnitude and phase variations in the healed myocardium are a good estimation of measurement errors. In our case, the measurement errors for the nontransmural measurements are in the range of $1.3 \Omega \cdot \mathrm{cm}$ and $0.98^{\circ}$ at $1 \mathrm{kHz}$. For the transmural measurements, the error is in the range of $5.1 \Omega \cdot \mathrm{cm}$ and $2.4^{\circ}$ throughout the frequency range. Note that the error, especially for the transmural measurements, is higher at low $\left(6.8 \Omega \cdot \mathrm{cm}\right.$ and $5.2^{\circ}$ at $\left.1 \mathrm{kHz}\right)$ and high frequencies $(10$ $\Omega \cdot \mathrm{cm}$ and $1.5^{\circ}$ at $562 \mathrm{kHz}$ ). This is due to the impedance of the reference electrode and the parasitic capacitances between the catheter and the body.

For healthy tissue, the transmural phase angle is greater than the nontransmural phase angle. This could be explained by taking into account the electrical current path across the tissue. In nontransmural measurements, the current mainly flows along the fiber direction, crossing fewer cell membranes than when it flows in a transmural direction.

Since there are more gap junctions in the longitudinal direction $(80 \%)$ than in the transversal direction (20\%) [20], the closing of gap junctions during acute ischemia will produce different impedance changes in the two directions. This effect can be seen in the results comparing the transmural spectrum with the nontransmural spectrum after $60 \mathrm{~min}$ of acute ischemia (Fig. 3). The low relaxation frequency in the nontransmural measurements $(10 \mathrm{kHz})$ does not appear in the transmural measurements. This result is in agreement with the work by Schaefer et al. [21]. Using nontransmural measurements, they detected a maximum increase of permittivity and a decrease of conductivity at $13 \mathrm{kHz}$ produced by the closing of gap junctions induced by the application of heptanol in the cardioplegic perfusion.

The magnitude of the impedance measured at $1 \mathrm{kHz}$ is adequate to differentiate between the three tissue states using a rigid four-needle probe with a well-known cell factor. However, using a three-electrode catheter method, the measured impedance is dependent not only on the specific impedance but also on the myocardium wall thickness. The changes in wall thickness in different parts of the heart and the intersubject variability will produce a bigger dispersion on the measured impedance. This could be seen in our results in the standard deviation (STD) for the transmural magnitude compared with the STD for the nontransmural magnitude (see Table I). For this reason, the differentiation in transmural measurements is better when the phase angle is used at selected frequencies.

We have shown previously [11] that it is possible to detect a healed transmural infarction using a percutaneous electrocatheter technique measuring the phase angle. Based on the results of the present study, it also seems feasible to detect acute ischemia during cardiac catheterization. Further work should include measuring the impedance spectrum for other cardiac tissues, for example, valvular, connective, and myocardial tissue damaged by RF ablation.

\section{CONCLUSION}

The electrical impedance spectrum of the myocardium depends on the state of the myocardium tissue, the current orientation respect to myocardial fibers, and the measurement technique. Accurate modeling of the myocardium must make use of anisotropic specific impedance or at least the most appropriate impedance value depending on the main current direction with respect to fiber orientation.

The magnitude of the specific impedance is similar for healthy myocardium regardless of the measurement method (transmural or nontransmural). However, the phase angle spectra for healthy myocardium, and especially for acute ischemic myocardium, were significantly different between both methods. Healed myocardial tissue showed a small and flat phase angle spectrum in both methods due to the low content of cells in the transmural infarct scar.

Using a four-needle electrode, the differentiation between tissue states is possible using the impedance magnitude at $1 \mathrm{kHz}$. When using a transmural method the measured impedance magnitude has a bigger dispersion due to its dependency with the wall thickness, reducing the separation between tissue states.

The impedance component that best distinguishes between tissue states for both methods is the phase angle; particularly for transmural measurements, the best measurement frequencies will be about 42 and $178 \mathrm{kHz}$. Therefore, phase spectrum must be considered the best option for differentiating myocardial tissue states for new impedance techniques using transcatheter methods.

\section{ACKNOWLEDGMENT}

The authors would like to thank the anonymous reviewers for their helpful comments and suggestions to improve the paper.

\section{REFERENCES}

[1] D. D. Streeter, H. M. Spotnitz, D. P. Patel, J. Ross Jr., and E. H. Sonnenblick, "Fiber orientation in the canine left ventricle during diastole and systole," Circ. Res., vol. 24, pp. 339-347, 1969.

[2] M. A. Fallert, M. S. Mirotznik, S. W. Downing, E. B. Savage, K. R. Foster, M. E. Josephson, and D. K. Bogen, "Myocardial electrical impedance mapping of ischemic sheep hearts and healing aneurysms," Circulation, vol. 87, pp. 199-207, 1993.

[3] S. Rush, J. A. Abildskov, and R. McFee, "Resistivity of body tissues at low frequencies," Circ. Res., vol. 12, pp. 40-50, 1963.

[4] D. E. Roberts and A. M. Scher, "Effect of tissue anisotropy on extracellular potential fields in canine myocardium in situ," Circ. Res., vol. 50, pp. 342-351, 1982.

[5] P. Steendijk, G. Mur, E. T. Van Der Velde, and J. Baan, "The four-electrode resistivity technique in anisotropic media: Theoretical analysis and application on myocardial tissue in vivo," IEEE Trans. Biomed. Eng., vol. 40, pp. 1138-48, Nov. 1993.

[6] J. Z. Tsai, J. A. Will, S. Hubbard-Van Stelle, H. Cao, S. Tungjitkusolmun, Y. B. Choy, D. Haemmerich, V. R. Vorperian, and J. G. Webster, "In vivo measurement of swine myocardial resistivity," IEEE Trans. Biomed. Eng., vol. 49, pp. 472-83, May 2002.

[7] S. Tungjitkusolmun, E. J. Woo, H. Cao, J. Z. Tsai, V. R. Vorperian, and J. G. Webster, "Thermal-electrical finite element modeling for radio frequency cardiac ablation: Effects of changes in myocardial properties," Med. Biol. Eng. Comput., vol. 38, no. 5, pp. 562-568, Sept. 2000. 
[8] H. Cao, S. Tungjitkusolmun, Y. B. Choy, J. Z. Tsai, V. R. Vorperian, and J. G. Webster, "Using electrical impedance to predict catheter-endocardial contact during RF cardiac ablation," IEEE Trans. Biomed. Eng., vol. 49, pp. 247-253, Mar. 2002.

[9] Y. Wang, D. R. Haynor, and Y. Kim, "A finite-element study of the effects of electrode position on the measured impedance change in impedance cardiography," IEEE Trans. Biomed. Eng., vol. 48, pp. 1390-1400, Dec. 2001

[10] D. Schwartzman, I. Chang, J. J. Michele, M. S. Mirotznik, and K. R. Foster, "Electrical impedance properties of normal and chronically infarcted left ventricular myocardium," J. Interv. Card. Electrophysiol., vol. 3, pp. 213-224, Oct. 1999.

[11] M. Warren, R. Bragos, O. Casas, A. Rodríguez-Sinovas, J. Rosell, I. Anivarro, and J. Cinca, "Percutaneous electrocatheter technique for on-line detection of healed transmural myocardial infarction," PACE, vol. 23, pp. 1283-1287, 2000

[12] X. Zheng, G. P. Walcott, J. A. Hall, D. L. Rollins, W. M. Smith, G. N. Kay, and R. E. Ideker, "Electrode impedance: An indicator of electrodetissue contact and lesion dimensions during linear ablation," J. Interv. Card. Electrophysiol., vol. 4, no. 4, pp. 645-54, Dec. 2000.

[13] D. Sheng He, M. Bosnos, M. Z. Mays, and F. Marcus, "Assessment of myocardial lesion size during in vitro radio frequency catheter ablation," IEEE Trans. Biomed. Eng., vol. 50, pp. 768-776, June 2003.

[14] R. Bragos, P. J. Riu, M. Warren, M. Tresanchez, A. Carreño, and J. Cinca, "Changes in myocardial impedance spectrum during acute ischemia in the in-situ pig heart," in Proc. 18th Annu. Int. Conf. Eng. Med. and Biol. Soc., vol. 5, Amsterdam, The Netherlands, 1996, pp. 1953-1954.

[15] J. Cinca, M. Warren, A. Rodríguez-Sinovas, M. Tresànchez, A. Carreño, R. Bragós, O. Casas, A. Domingo, and J. Soler-Soler, "Passive transmission of ischemic ST segment changes in low electrical resistance myocardial infarct scar in the pig," Cardiovasc. Res., vol. 40, pp. 103-112, 1998.

[16] M. I. Ellenby, K. W. Small, R. M. Wells, D. J. Hoyt, and J. E. Lowe, "On-line detection of reversible myocardial ischemic injury by measurement of myocardial electrical impedance," Ann. Thorac. Surg., vol. 44, pp. 587-597, 1987.

[17] J. Cinca, M. Warren, A. Carreño, M. Tresànchez, L. Armadans, P. Gómez, and J. Soler-Soler, "Changes in myocardial electrical impedance induced by coronary artery occlusion in pigs with and without preconditioning," Circulation, vol. 96, pp. 3079-3086, 1997.

[18] R. Bragos, O. Casas, J. Rosell, P. J. Riu, A. Rodriguez-Sinovas, and J. Cinca, "Detection of healed myocardium scar in pigs measuring the electrical impedance spectrum with a percutaneous catheter," in Proc. XI Int. Conf. Electrical Bio. Impedance, Oslo, Norway, 2001, pp. 63-66.

[19] R. Bragós, P. J. Riu, J. Cairó, J. L. Montesinos, and A. Tintó, "On-line estimation of biomass in free and immobilized cell cultures using two-frequency measurements," in Proc. IX Int. Conf. Electrical Bioimpedance, Heidelberg, Germany, 1995, pp. 59-62.

[20] R. H. Hoyt, M. L. Cohen, and J. E. Saffitz, "Distribution and three-dimensional structure of intercellular junctions in canine myocardium," Circ. Res., vol. 64, pp. 563-574, 1989.

[21] M. Schaefer, W. Gross, J. Ackmann, and M. M. Gebhard, "The complex dielectric spectrum of heart tissue during ischemia," Bioelectrochemistry, vol. 58, pp. 171-180, 2002.

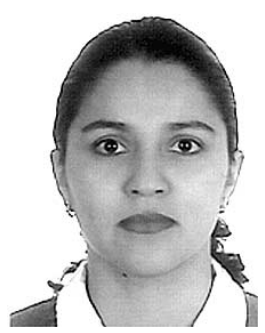

Yolocuauhtli Salazar was born in Durango, Mexico, in February 1978. She received the Ingeniero Electrónico degree from the Instituto Tecnológico de Durango, México, in 2000. She is currently working toward the Ph.D. degree at the Universidad Politécnica de Cataluña, Barcelona, Spain.

Her research interests include electrical impedance spectroscopy, finite element modeling, and myocardial characterization.

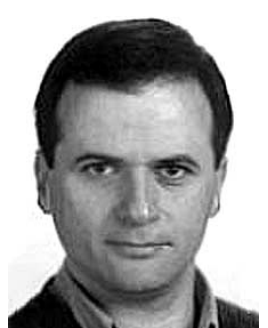

Ramon Bragos (S'90-M'94) received the telecommunication engineering degree and the Eng.D. degree from the Universidad Politécnica de Cataluña (UPC), Barcelona, Spain, in 1991 and 1997, respectively.

From 1991 to 1998, he was an Assistant Professor with the Electronics Engineering Department, UPC, where he is currently is an Associate Professor. He is a member of the Biomedical Research Center at the UPC. His research interest includes biomedical and biotechnological instrumentation techniques, in particular noninvasive techniques based on electrical impedance spectroscopy.

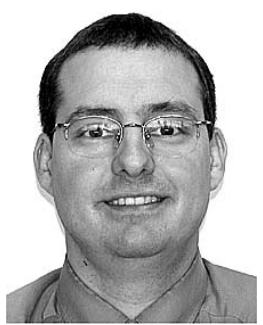

Oscar Casas (S'93-A'99) received the Ingeniero de Telecomunicación and Doctor Ingeniero de Telecomunicación degrees from the Universitat Politécnica de Cataluña (UPC), Barcelona, Spain, in 1994 and 1998 , respectively.

In 1994, he joined the Department of Electronic Engineering, UPC, as a Lecturer and, since 2001, he has been an Associate Professor, teaching courses in several areas of electronic instrumentation. His research interests include biomedical and electronic instrumentation.

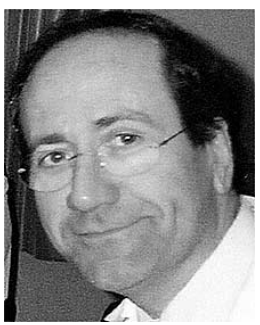

Juan Cinca is Professor of Medicine and Chief of the Department of Cardiology, University Hospital Sant Pau, Barcelona, Spain

His research interests include clinical and basic electrophysiology and pathophysiology of ischemic heart diseases.

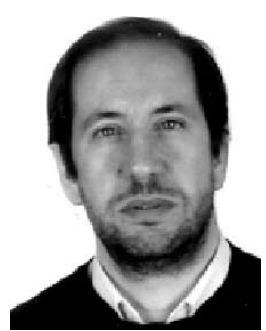

Javier Rosell (S'84-M'89) was born in Barcelona, Spain, in June 1959. He received the Ingeniero de Telecomunicación and Doctor Ingeniero de Telecomunicación degrees from the Polytechnic University of Cataluña (UPC), Barcelona, Spain, in 1983 and 1989, respectively.

$\mathrm{He}$ is currently a Professor with the Department of Electronic Engineering, UPC, and is also with the Biomedical Research Center, UPC where he is the head of the Biomedical Instrumentation group. His current research interests are focused in noninvasive measurement methods in the medical and biological fields, in particular, based on electrical impedance tomography and magnetic induction spectroscopy. 